\title{
HbA1c-Based Score Model for Predicting Death Risk in Patients with Hepatocellular Carcinoma and Type 2 Diabetes Mellitus
}

\author{
Lingling He, Shuan Zhang, Xiaoli Liu, Yuyong Jiang, Xianbo Wang, and Zhiyun Yang \\ Department of Traditional Chinese Medicine, Beijing Ditan Hospital and Collaborative Innovation Center of Infectious Diseases, \\ Capital Medical University, Chaoyang, Beijing 100015, China
}

Correspondence should be addressed to Zhiyun Yang; yangzhiyun66@163.com

Received 11 November 2016; Revised 28 November 2016; Accepted 12 December 2016; Published 5 January 2017

Academic Editor: Chun Gao

Copyright (C) 2017 Lingling He et al. This is an open access article distributed under the Creative Commons Attribution License, which permits unrestricted use, distribution, and reproduction in any medium, provided the original work is properly cited.

Aim. To establish a new score model to predict risk of death in patients with hepatocellular carcinoma and type 2 diabetes mellitus. Methods. This was a retrospective study of 147 patients with hepatocellular carcinoma and type 2 diabetes mellitus who came to Beijing Ditan Hospital between October 2008 and June 2013. Univariate and multivariate logistic regression analysis was performed to obtain the independent factors associated with death risk. A new score model was devised according to these factors. Results. A prediction score model composed of HbAlc, NLR, age, and CTP class was devised, which ranged from 0 to 7 . AUROC of the score was $0.853(P<0.001,95 \%$ CI: 0.791-0.915). Scores 0-2, 3-4, and 5-7 identified patients as low-, medium-, and high-risk categories. The cumulative survival rate was $93.6 \%, 83.0 \%$, and $74.5 \%$ in the low-risk group in 1,2 , and 3 years, while it was $64.0 \%, 46.0 \%$, and $26.0 \%$ in the medium-risk group, whereas it was $24.0 \%, 12.0 \%$, and $6.0 \%$ in the high-risk group, respectively. The cumulative survival rate was significantly higher in the low-risk group than that in the medium-risk group and high-risk group $(P<0.001)$. Conclusion. The HbAlc-based score model can be used to predict death risk in patients with hepatocellular carcinoma and type 2 diabetes mellitus.

\section{Introduction}

Liver cancer is the second leading cause of cancer death worldwide in men and the most primary liver cancer is hepatocellular carcinoma (HCC) [1]. It is reported that the overall median survival time was only 6 months in patients with HCC [2]. Clinical data indicate that type 2 diabetes mellitus (T2DM) is associated with the incidence and development of hepatocellular carcinoma [3-6].

A series of score models have been established to predict risk of HCC in chronic liver diseases [7-11]. However, a score model for predicting death risk is badly in need, especially in patients with HCC and T2DM.

In this retrospective cohort study, we analyzed the baseline clinical data of patients with HCC and T2DM. We aimed to identify the independent risk factors in these patients, to establish a score model for predicting death risk, and to stratify patients for the level of care they need.

\section{Patients and Method}

2.1. Study Population. This was a retrospective cohort study. During the period between October 2008 and June 2013, all patients with hepatocellular carcinoma and type 2 diabetes who were first seen in Beijing Ditan Hospital, Capital Medical University, China, were followed up for the risk of death in three years. Patients who had metastatic cancer of liver, other serious concurrent illnesses, and incomplete clinical data were excluded.

2.2. Clinical and Laboratory Indicators. The baseline indicators were collected including a full medical history, the clinical indicators, and laboratory indexes like white blood cell (WBC), neutrophil-lymphocyte ratio (NLR), platelet (PLT), hemoglobin (HGB), liver and renal biochemistries, total cholesterol (TC), high density lipoprotein cholesterol (HDLC), glycosylated hemoglobin (HbAlc), prothrombin time (PT), and alpha fetoprotein (AFP). The transabdominal 
TABLE 1: Characteristics of patients.

\begin{tabular}{|c|c|c|c|c|}
\hline Variable & $\begin{array}{l}\text { Total patients } \\
\quad(N=147)\end{array}$ & $\begin{array}{l}\text { Survival group } \\
\quad(N=51)\end{array}$ & $\begin{array}{c}\text { Death group } \\
\quad N=96\end{array}$ & $P$ value \\
\hline Age (yr) & $58.93 \pm 9.50$ & $56.80 \pm 10.15$ & $60.06 \pm 8.98$ & 0.050 \\
\hline Male sex & $116(78.9)$ & $42(82.4)$ & 74 (77.1) & 0.456 \\
\hline History of smoking & $57(40.1)$ & $23(45.1)$ & $34(35.4)$ & 0.288 \\
\hline History of alcohol use & $47(32.0)$ & $16(31.4)$ & $31(32.3)$ & 1.000 \\
\hline Family history of liver disease & $35(23.8)$ & $11(21.6)$ & $24(25.0)$ & 0.689 \\
\hline Family history of diabetes & $6(4.1)$ & $3(5.9)$ & $3(3.1)$ & 0.418 \\
\hline $\mathrm{WBC}\left(\times 10^{9} / \mathrm{L}\right)$ & $5.0 \pm 2.7$ & $4.4 \pm 2.1$ & $5.3 \pm 2.9$ & $<0.001$ \\
\hline NLR & $3.0(1.8,5.3)$ & $1.9(1.5,3.1)$ & $4.1(2.1,6.7)$ & $<0.001$ \\
\hline $\operatorname{PLT}\left(\times 10^{9} / \mathrm{L}\right)$ & $79.8(53.4,148.9)$ & $83.0(59.0,122.3)$ & $79.2(50.8,156.8)$ & 0.880 \\
\hline $\operatorname{HGB}(g / l)$ & $116.1 \pm 23.5$ & $123.9 \pm 24.0$ & $112.0 \pm 22.3$ & $<0.001$ \\
\hline $\operatorname{ALT}(\mathrm{U} / \mathrm{L})$ & $33.7(22.5,55.1)$ & $32.6(21.5,50.5)$ & $34.3(25.2,59.4)$ & 0.490 \\
\hline TBIL $(\mu \mathrm{mol} / \mathrm{l})$ & $21.9(12.3,42.1)$ & $15.8(9.7,26.3)$ & $29.6(15.2,64.6)$ & $<0.001$ \\
\hline GGT (U/L) & $70.5(34.0,146.2)$ & $49.5(29.0,96.3)$ & $86.5(42.3,198.5)$ & $<0.001$ \\
\hline $\operatorname{ALB}(g / l)$ & $33.7 \pm 6.3$ & $37.2 \pm 6.0$ & $31.9 \pm 5.6$ & $<0.001$ \\
\hline $\mathrm{TC}(\mathrm{mmol} / \mathrm{L})$ & $3.5(2.8,4.2)$ & $3.8(3.0,4.2)$ & $3.3(2.8,4.1)$ & 0.170 \\
\hline HDLC (mmol/L) & $1.0 \pm 0.5$ & $1.2 \pm 0.4$ & $0.9 \pm 0.5$ & $<0.001$ \\
\hline $\operatorname{HbAlc}(\%)$ & $7.0 \pm 2.0$ & $6.4 \pm 1.9$ & $7.4 \pm 2.0$ & 0.010 \\
\hline $\mathrm{Cr}(\mu \mathrm{mol} / \mathrm{l})$ & $64.0(54.0,77.0)$ & $62.0(53.0,71.0)$ & $66.0(54.3,85.3)$ & 0.040 \\
\hline $\mathrm{PT}(\mathrm{s})$ & $14.1 \pm 2.8$ & $13.4 \pm 2.7$ & $14.5 \pm 2.8$ & $<0.001$ \\
\hline $\operatorname{AFP}(n g / m l)$ & $15.2(4.7,169.2)$ & $16.8(4.6,83.3)$ & $15.1(5.5,280.8)$ & 0.390 \\
\hline MELD score & $22.4 \pm 7.0$ & $19.8 \pm 5.6$ & $23.8 \pm 7.3$ & $<0.001$ \\
\hline CTP class $(\mathrm{A} / \mathrm{B} / \mathrm{C})$ & $53 / 51 / 43$ & $31 / 12 / 8$ & $22 / 39 / 35$ & $<0.001$ \\
\hline
\end{tabular}

ultrasonography, computed tomography, and nuclear magnetic resonance imaging were also collected.

2.3. The Diagnosis of HCC. The diagnosis of HCC was mostly based on histopathological confirmation. Some patients were included by clinical diagnosis, which mean a positive lesion was detected with at least two imaging techniques (hepatic arteriography, nuclear magnetic resonance imaging, computed tomography, or abdominal ultrasonography) or detected with one imaging technique coupled with AFP level above $400 \mathrm{ng} / \mathrm{ml}$ [12].

2.4. Statistical Analysis. Statistical analysis was performed by SPSS version 19.0 (IBM Corp., Armonk, NY, USA). Quantitative data accorded with normal distribution were expressed in mean \pm standard deviation and they were analyzed by Student's $t$-test. Data not accorded with normal distribution were expressed in median with interquartile range and they were analyzed by Mann-Whitney U test. Qualitative data were analyzed by chi-square test. Univariate and multivariate logistic regression analysis was performed to determine the variables associated with the prognosis. The risks were expressed in odd ratio (OR) and 95\% confidence interval (CI). The regression coefficients were converted to integer risk scores and the final score was the sum of these values. The Kaplan-Meier method was used to estimate the cumulative survival of different groups. The log-rank test was used to compare time-to-event curves between different groups. The diagnostic value of the new score model was estimated by areas under the receiver operating characteristics (AUROC) curve. $P<0.05$ was considered statistically significant.

\section{Results}

3.1. Patient Characteristics. During the study period, a total of 147 patients were included in the study. At a median followup time of 21 months, 96 (65.3\%) of 147 patients died in three years. The 1,2 , and 3 years' cumulative survival rates were $59.9 \%, 46.3 \%$, and $34.7 \%$, respectively. The baseline characteristics of patients were shown in Table 1. Patients who died in three years were older and had higher WBC, higher NLR, lower HGB, higher total bilirubin (TBIL), higher gamma-glutamyl transpeptidase (GGT), lower serum albumin (ALB), higher creatinine (Cr), lower HDLC, higher HbAlc, longer PT, higher MELD score, and a fairly large proportion of patients with CTP class C patients (Table 1).

3.2. Factors Associated with Death Risk. Table 2 shows the results of univariate and multivariate logistic regression analysis. By univariate analysis, age, WBC, NLR, HGB, HbAlc, GGT, MELD score, and CTP class were found to be significant risk factors for the incidence of death. By multivariate analysis, age, NLR, HbAlc, and CTP class 
TABLE 2: Factors associated with death risk in patients with HCC and T2DM.

\begin{tabular}{|c|c|c|c|c|c|c|}
\hline \multirow{2}{*}{ Variable } & \multicolumn{3}{|c|}{ Univariate } & \multicolumn{3}{|c|}{ Multivariable } \\
\hline & $P$ value & Odds ratio & $95 \% \mathrm{CI}$ & $P$ value & Odds ratio & $95 \% \mathrm{CI}$ \\
\hline Age & 0.050 & 1.038 & $1.000-1.078$ & 0.043 & 1.048 & $1.001-1.096$ \\
\hline WBC & 0.049 & 1.161 & $1.001-1.346$ & & & \\
\hline NLR & $<0.001$ & 1.398 & $1.168-1.673$ & 0.015 & 1.258 & $1.046-1.514$ \\
\hline HGB & 0.005 & 0.977 & $0.962-0.993$ & & & \\
\hline HbAlc & 0.006 & 1.315 & $1.080-1.602$ & 0.005 & 1.362 & $1.096-1.693$ \\
\hline GGT & 0.011 & 1.005 & $1.001-1.009$ & & & \\
\hline MELD score & 0.001 & 1.101 & $1.038-1.168$ & & & \\
\hline CTP class & $<0.001$ & 2.714 & $1.668-4.414$ & 0.006 & 1.312 & $1.083-1.590$ \\
\hline
\end{tabular}

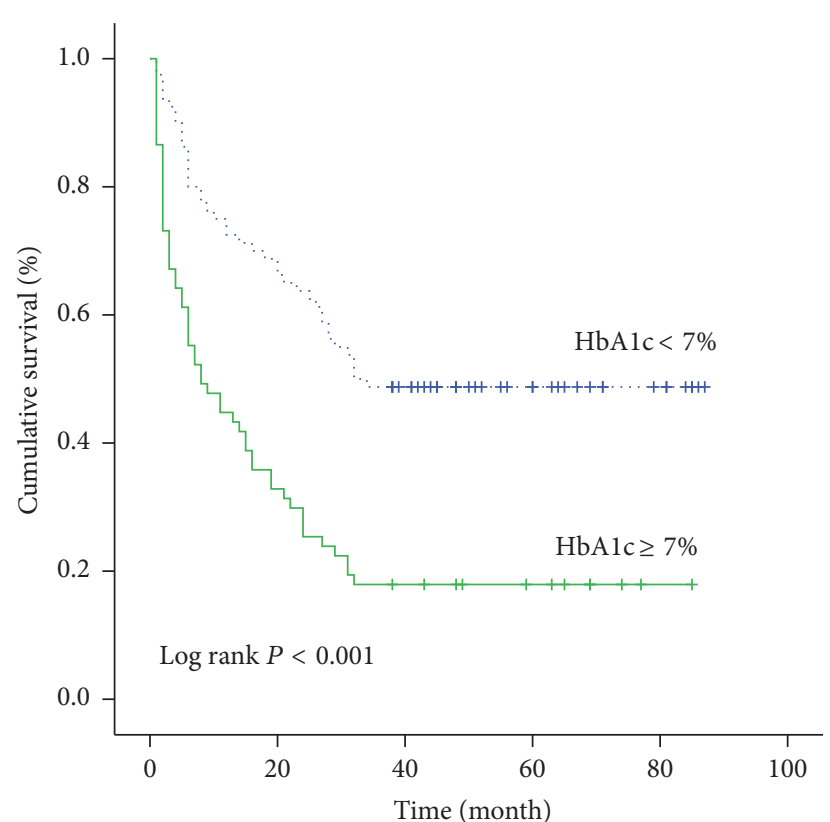

FIGURE 1: The cumulative survival rate of patients in stratified HbAlc groups.

remained the independent risk factors associated with high risk of mortality.

A further analysis was performed to evaluate the influence of each risk factor on the cumulative survival rate. The 3year cumulative survival rate was $48.8 \%$ in $\mathrm{HbAlc}<7 \%$ group while it was $17.9 \%$ in $\mathrm{HbAlc} \geq 7 \%$ group (Figure 1). It was significantly higher in HbAlc $<7 \%$ group than that in HbAlc $\geq 7 \%$ group. Similarly, the 3 -year cumulative survival rate was $52.5 \%$ in the NLR $\leq 3.27$ group while it was $13.4 \%$ in the NLR $>3.27$ group (Figure 2). The 3-year cumulative survival rate was $45.3 \%$ in the age $\leq 56$ year group while it was $26.5 \%$ in the age $>56$ year group (Figure 3 ). The 3 -year cumulative survival rate was $58.5 \%$ in CTP class A group, whereas it was $23.5 \%$ in CTP class B group and 18.6\% in CTP class C group (Figure 4). It was significantly higher in CTP class A group than that in CTP class B group and CTP class C group.

3.3. Prediction Score Model. A new score model was devised using independent risk factors in the multivariate logistic

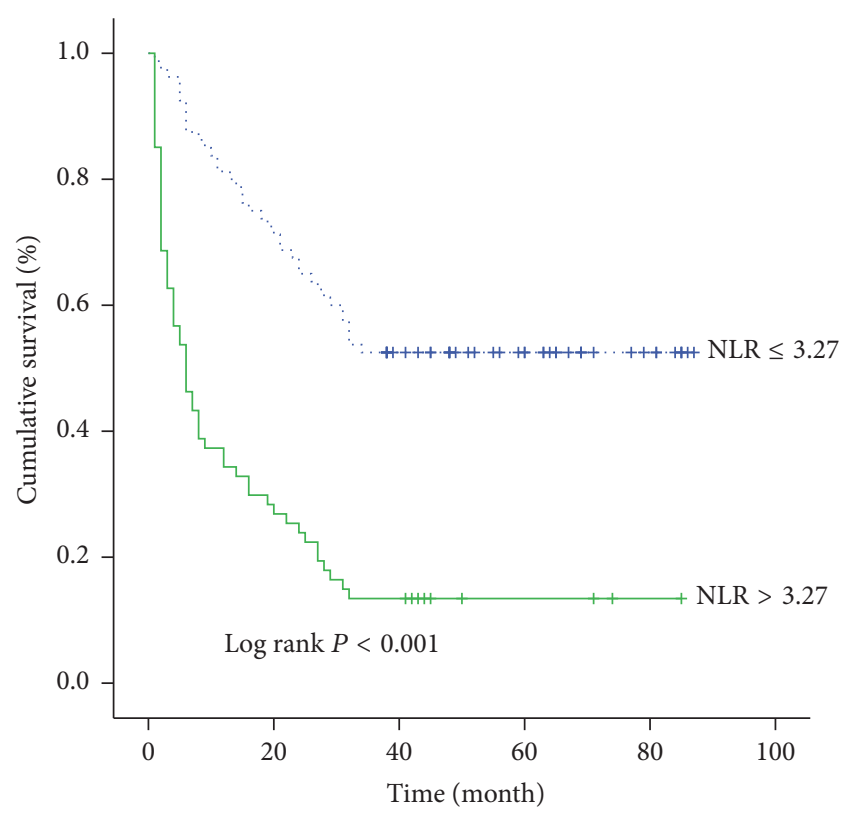

FIgURE 2: The cumulative survival rate of patients in stratified NLR groups.

TABLE 3: Components of the score model.

\begin{tabular}{lcccc}
\hline Variable & $\begin{array}{c}\text { Regression } \\
\text { coefficient }\end{array}$ & $P$ value & Odds ratio & $\begin{array}{c}\text { Risk } \\
\text { score }\end{array}$ \\
\hline Age $\geq 57(\mathrm{yr})$ & 1.317 & 0.005 & 3.734 & 1 \\
$\mathrm{HbAlc} \geq 7(\%)$ & 2.195 & $<0.001$ & 8.976 & 2 \\
NLR $\geq 3.27$ & 1.905 & $<0.001$ & 6.72 & 2 \\
CTP class A & & & & \\
CTP class B & 1.734 & 0.001 & 5.662 & 2 \\
CTP class C & 1.717 & 0.004 & 5.567 & 2 \\
\hline
\end{tabular}

regression analysis. It was attributed to each parameter according to its relative contribution, as determined by the regression coefficients (Table 3 ). The score ranged from 0 to 7 . AUROC was $0.853(P<0.001,95 \%$ CI: $0.791-0.915)$ (Figure 5). We observed that the survival rate gradually decreased with increasing score, from $87.5 \%$ in score $0,73.3 \%$ 


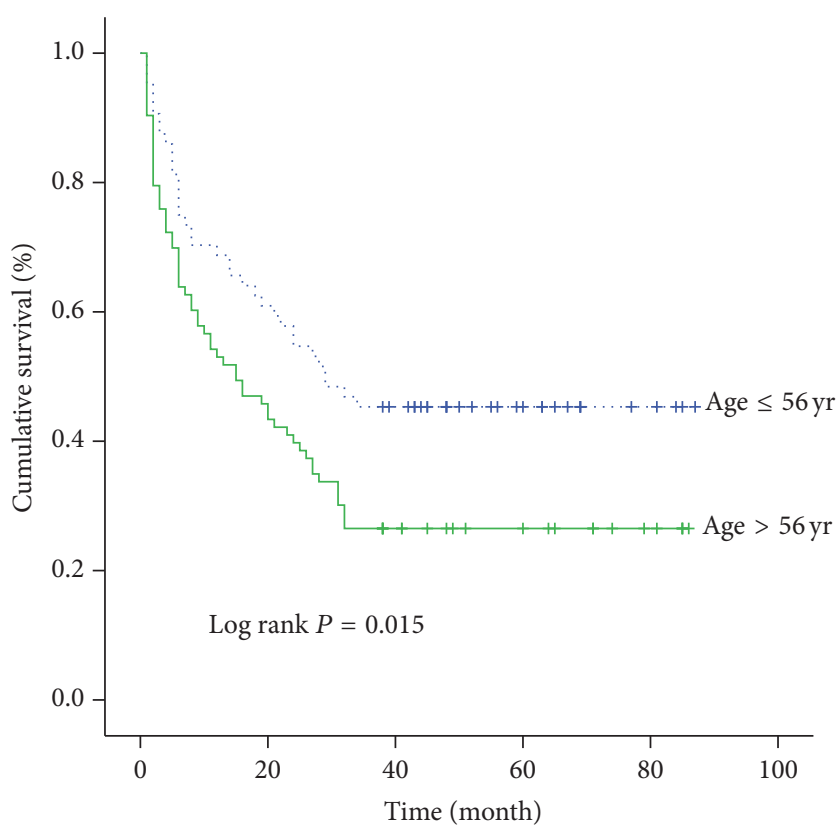

FIgURE 3: The cumulative survival rate of patients in stratified age groups.

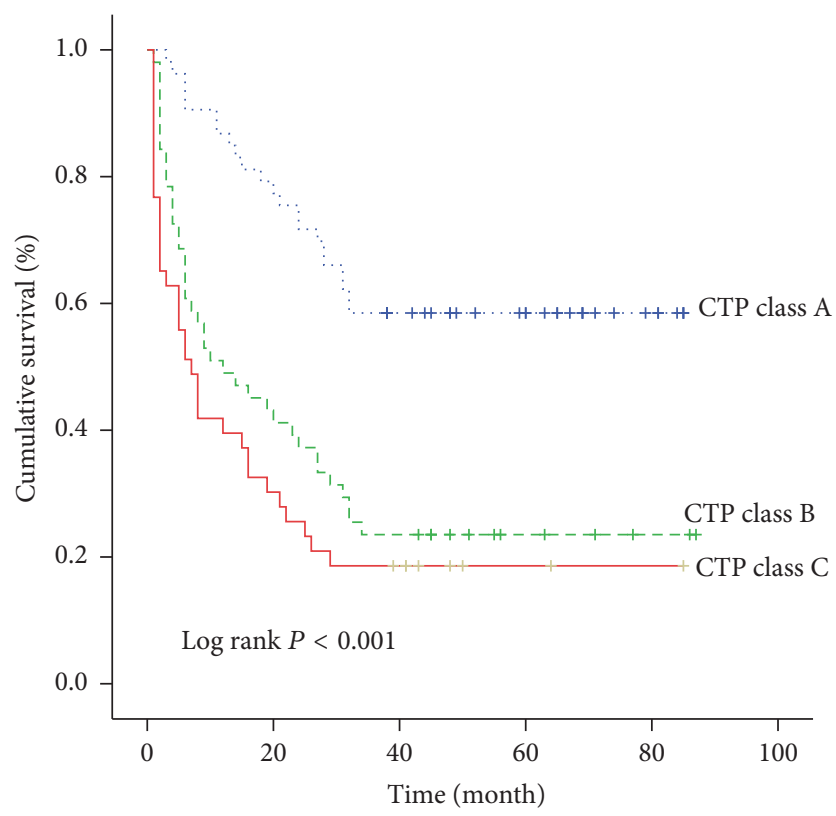

FIgURE 4: The cumulative survival rate of patients in different CTP class.

in score $1,70.8 \%$ in score $2,30.8 \%$ in score $3,20.8 \%$ in score 4 , and $14.3 \%$ in score 5 to $0.0 \%$ in scores 6 and 7 (Figure $6(\mathrm{a})$ ).

We assigned the scores $0-2,3-4$, and 5-7 to low-, medium-, and high-risk categories; thus, 47, 50, and 50 patients belonged to each category, respectively. In the lowrisk group, 35 (74.5\%) patients survived in three years after they were diagnosed with HCC and T2DM. Correspondingly, the patients who survived in the medium-risk group and

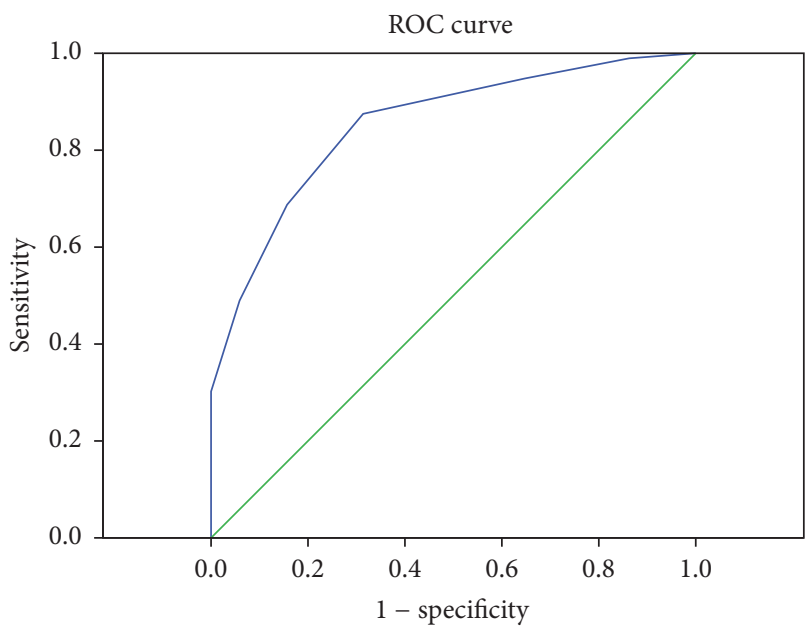

FIgURE 5: AUROC of HbAlc-based score model.

TABLE 4: Death risk in stratified score groups.

\begin{tabular}{lcccc}
\hline Score & $\begin{array}{c}\text { Regression } \\
\text { coefficient }\end{array}$ & $P$ value & Odds ratio & $95 \%$ CI \\
\hline $0-2$ & & & Reference & \\
$3-4$ & 2.116 & $<0.001$ & 8.301 & $3.339-20.635$ \\
$5-7$ & 3.822 & $<0.001$ & 45.694 & $11.981-174.281$ \\
\hline
\end{tabular}

high-risk group were $13(26.0 \%)$ and $3(6.0 \%)$, respectively (Figure 6(b)).

The cumulative survival rate was $93.6 \%, 83.0 \%$, and $74.5 \%$ in the low-risk group in 1, 2, and 3 years, while it was $64.0 \%$, $46.0 \%$, and $26.0 \%$ in the medium-risk group, whereas it was $24.0 \%, 12.0 \%$, and $6.0 \%$ in the high-risk group, respectively. The cumulative survival rate was significantly higher in the low-risk group than that in the medium-risk group and highrisk group $(P<0.001)$ (Figure 7$)$. We also observed that the death risk in low-, medium-, and high-risk categories was increased significantly because the odds ratio of these catigories increased multiply (Table 4).

\section{Discussion}

In this study, we established the risk score model based on $\mathrm{HbAlc}$ to predict death risk in patients with hepatocellular carcinoma and type 2 diabetes mellitus. This model was not only accurate and sensitive to predict 3-year mortality, but also simple and easy to be applied in the clinical practice as it was composed of four routinely available clinical and laboratory parameters. Patients in different risk group had distinctly different risk of death. The 3-year cumulative survival rate was $74.5 \%$ in the low-risk group while it was just $6.0 \%$ in the high-risk group. Predicting the risk of death accurately could let clinicians pay more attention to the high-risk group and give them more appropriate medical care. With this method, the health care resources could be allocated and utilized more efficiently.

There have been various kinds of risk models to predict HCC in chronic liver diseases [7-11]. In several models, 


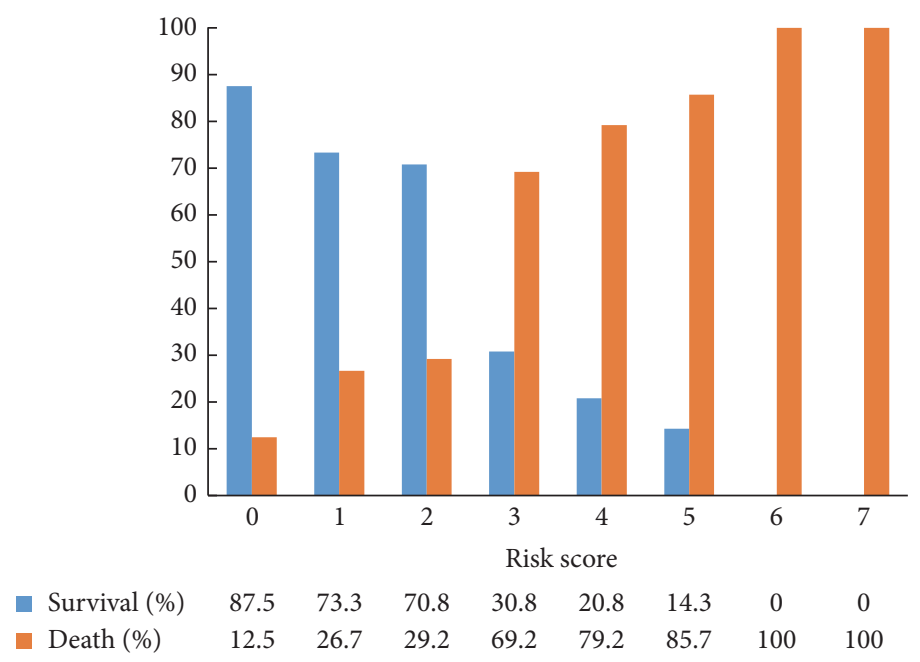

(a)

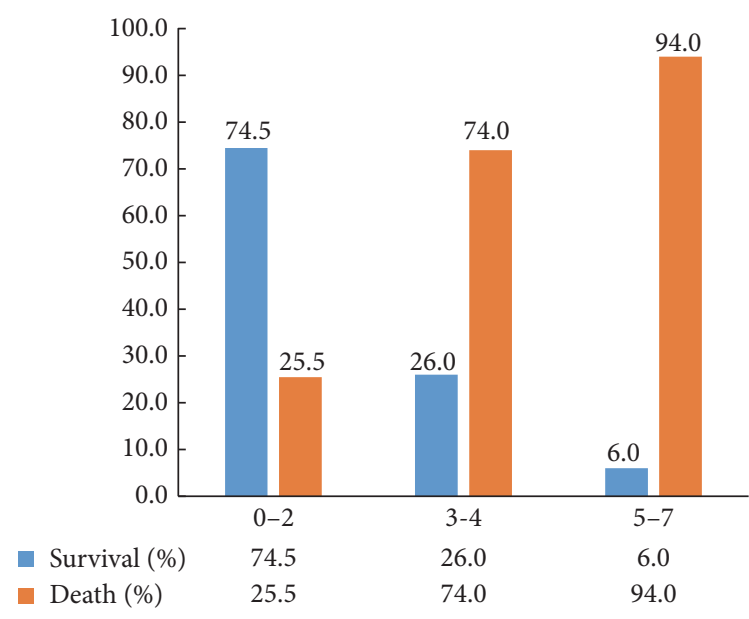

(b)

FIgURE 6: (a) The survival and mortality rate of patients in each score. (b) The survival and mortality rate of patients in stratified score groups.

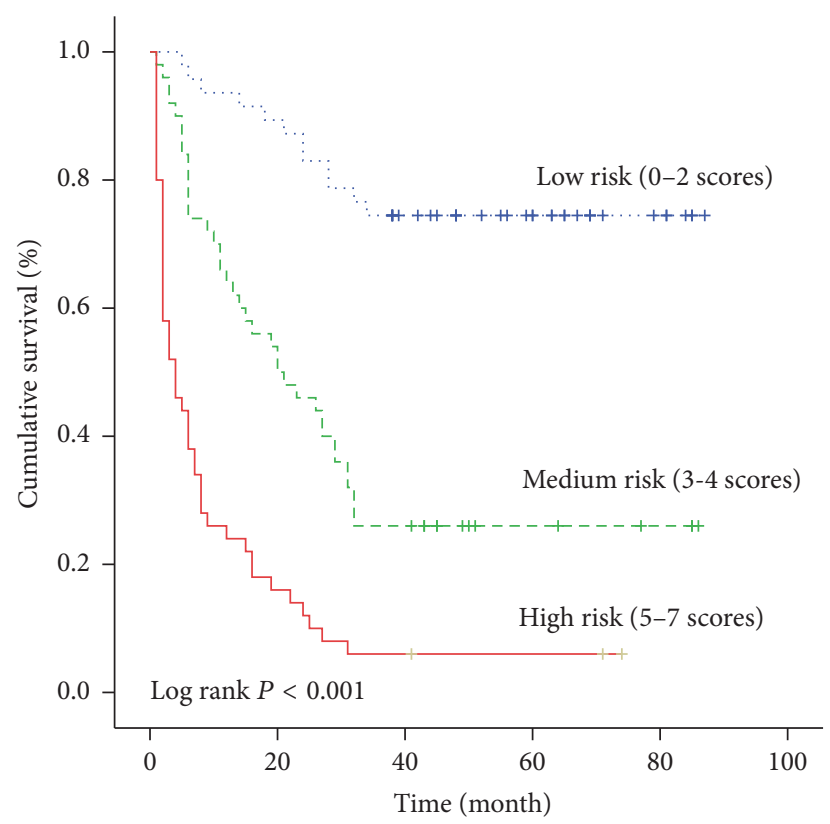

Figure 7: The cumulative survival rate of patients in different risk groups.

diabetes is one of the variables [10,11]. It is also reported that history of diabetes is associated with deaths from HCC [13]. Patients with DM showed significantly lower overall survival than those without DM $[14,15]$. Thus, a specific group of people with HCC and T2DM should be observed. To our knowledge, this is the first study to establish a prediction score model for death risk in the specific population of HCC and T2DM.

HbAlc is a biomarker of the blood glucose level over the last 3 months and it is believed to be the robust indicator of diabetes management. Recent studies have shown that increased serum $\mathrm{HbAlc}$ level is associated with the development and progression of cancer [16]. Furthermore, higher HbAlc level is correlated with higher risk of all-cause mortality in cancer patients [17-20]. In pancreatic cancer patients with diabetes, survival time of $\mathrm{HbAlc} \geq 7.0 \%$ group was significantly higher than that of HbAlc $<7.0 \%$ group [21]. The mortality risk was twice as high in breast cancer patients with $\mathrm{HbAlc} \geq 7.0 \%$ compared with $\mathrm{HbAlc}<6.5 \%$ patients [22]. In our study, HbAlc is the most important parameter in the risk score model. The median survival time in $\mathrm{HbAlc} \geq$ $7.0 \%$ group was 33 months compared with 8 months in HbAlc $<7.0 \%$ group. The risk of mortality was eight times higher in $\mathrm{HbAlc} \geq 7.0 \%$ group than that in HbAlc $<7.0 \%$ group (OR: 8.976, 95\% CI: 3.164-25.460). These results add to evidence that any clinical intervention should be put into practice to reduce serum $\mathrm{HbAlc}$ level to reduce mortality risk.

NLR has recently been reported to predict prognosis of HCC in several studies [23,24]. Higher NLR level was associated with poor disease-free survival and overall survival [2527]. Our study found that NLR $>3.27$ may be an indicator of poor overall survival in patients with HCC and T2DM. The median survival time in NLR > 3.27 group was 38 months, while it was only 6 months in NLR $\leq 3.27$ group.

Age is believed to be an independent factor associated with prognosis in most studies and it is an important parameter in a variety of score models [7-11]. In the present study, patients under age of 56 have longer median survival time than patients older than 56 ( 29 versus 15 months).

Numerous studies have compared the power of ChildTurcotte-Pugh class with MELD score in predicting prognosis in patients with liver disease [28-30]. The current study confirmed that CTP class was one of the independent risk factors in the multivariate analysis in patients with HCC and T2DM, whereas MELD score was not. Nevertheless, there is no significant difference between CTP class B group and CTP class $C$ group in terms of 3 -year cumulative survival rate $(P=0.188)$. Furthermore, the risk of mortality in CTP class 
$\mathrm{B}$ and $\mathrm{C}$ groups were nearly the same when compared to CTP class A group (OR: 5.662, 95\% CI: 1.961-16.343 and OR: 5.567, 95\% CI: 1.711-18.118).

The potential limitations of our study should be clarified. One is that the variables analyzed in this study were baseline covariates and the treatment strategy was not taken into account. More still needs to be done to optimize this score model that may trigger different clinical interventions. The other is that the population included in this study is small that a further validation of a large prospective cohort study is necessary to evaluate the prediction accuracy.

In conclusion, HbAlc, NLR, age, and CTP class are independently correlated with death risk of patients with HCC and T2DM. A novel score model based on HbAlc has been formulated and it is of great use to permit clinicians to identify the individual risk of death and to stratify these patients for the level of care they need.

\section{Competing Interests}

The authors declare that they have no competing interests.

\section{Authors' Contributions}

Lingling $\mathrm{He}$ and Shuan Zhang contributed equally to this research.

\section{Acknowledgments}

This study was supported by the Beijing Natural Science Foundation (Grant no. 7142081) (applicant: Zhiyun Yang), the Capital Health Research and Development of Special (Grant no. 2016-2-2171) (applicant: Zhiyun Yang), and the Science and Technology Project of Beijing Municipal Education Commission (Grant no. SQKM201610025026) (applicant: Zhiyun Yang).

\section{References}

[1] L. A. Torre, F. Bray, R. L. Siegel, J. Ferlay, J. Lortet-Tieulent, and A. Jemal, "Global cancer statistics, 2012," CA Cancer Journal for Clinicians, vol. 65, no. 2, pp. 87-108, 2015.

[2] B. Njei, Y. Rotman, I. Ditah, and J. K. Lim, "Emerging trends in hepatocellular carcinoma incidence and mortality," Hepatology, vol. 61, no. 1, pp. 191-199, 2015.

[3] S. Schlesinger, K. Aleksandrova, T. Pischon et al., "Diabetes mellitus, insulin treatment, diabetes duration, and risk of biliary tract cancer and hepatocellular carcinoma in a European Cohort," Annals of Oncology, vol. 24, no. 9, pp. 2449-2455, 2013.

[4] T. M. Welzel, B. I. Graubard, S. Quraishi et al., "Populationattributable fractions of risk factors for hepatocellular carcinoma in the United States," American Journal of Gastroenterology, vol. 108, no. 8, pp. 1314-1321, 2013.

[5] Z. Zheng, C. Zhang, J. Yan et al., "Diabetes mellitus is associated with hepatocellular carcinoma: a retrospective case-control study in hepatitis endemic area," PLoS ONE, vol. 8, no. 12, Article ID e84776, 2013.

[6] C. Gao, H.-C. Zhao, J.-T. Li, and S.-K. Yao, "Diabetes mellitus and hepatocellular carcinoma: comparison of Chinese patients with and without HBV-related cirrhosis," World Journal of Gastroenterology, vol. 16, no. 35, pp. 4467-4475, 2010.

[7] V. W.-S. Wong, S. L. Chan, F. Mo et al., "Clinical scoring system to predict hepatocellular carcinoma in chronic hepatitis B carriers," Journal of Clinical Oncology, vol. 28, no. 10, pp. 1660$1665,2010$.

[8] H. Yang, M. Yuen, H. L. Chan et al., "Risk estimation for hepatocellular carcinoma in chronic hepatitis B (REACH-B): development and validation of a predictive score," The Lancet Oncology, vol. 12, no. 6, pp. 568-574, 2011.

[9] G. L.-H. Wong, H. L.-Y. Chan, C. K.-Y. Wong et al., "Liver stiffness-based optimization of hepatocellular carcinoma risk score in patients with chronic hepatitis B," Journal of Hepatology, vol. 60, no. 2, pp. 339-345, 2014.

[10] T. Michikawa, M. Inoue, N. Sawada et al., "Development of a prediction model for 10-year risk of hepatocellular carcinoma in middle-aged Japanese: the Japan Public Health Center-Based Prospective Study Cohort II," Preventive Medicine, vol. 55, no. 2, pp. 137-143, 2012.

[11] J. A. Flemming, J. D. O. Yang, E. Vittinghoff, W. R. Kim, and N. A. Terrault, "Risk prediction of hepatocellular carcinoma in patients with cirrhosis: the ADRESS-HCC risk model," Cancer, vol. 120, no. 22, pp. 3485-3493, 2014.

[12] J. Bruix and M. Sherman, "Management of hepatocellular carcinoma: an update," Hepatology, vol. 53, no. 3, pp. 1020-1022, 2011.

[13] C.-H. Chiang, C.-W. Lu, H.-C. Han et al., "The relationship of diabetes and smoking status to hepatocellular carcinoma mortality," Medicine, vol. 95, no. 6, Article ID e2699, 2016.

[14] Y.-G. Wang, P. Wang, B. Wang, Z.-J. Fu, W.-J. Zhao, and S.-L. Yan, "Diabetes mellitus and poorer prognosis in hepatocellular carcinoma: a systematic review and meta-analysis," PLoS ONE, vol. 9, no. 5, Article ID e95485, 2014.

[15] Y.-Y. Wang, S. Huang, J.-H. Zhong et al., "Impact of diabetes mellitus on the prognosis of patients with hepatocellular carcinoma after curative hepatectomy," PLoS ONE, vol. 9, no. 12, Article ID el13858, 2014.

[16] M. Zhang, X. Li, X. Zhang, Y. Yang, Z. Feng, and X. Liu, "association of serum hemoglobin Alc, C-peptide and insulinlike growth factor-1 levels with the occurrence and development of lung cancer," Molecular and Clinical Oncology, vol. 2, no. 4, pp. 506-508, 2014.

[17] M. P. Bancks, A. O. Odegaard, J. S. Pankow et al., "Glycated hemoglobin and all-cause and cause-specific mortality in Singaporean Chinese without diagnosed diabetes: the Singapore Chinese Health Study," Diabetes Care, vol. 37, no. 12, pp. 31803187, 2014.

[18] K.-T. Khaw, N. Wareham, R. Luben et al., "Glycated haemoglobin, diabetes, and mortality in men in Norfolk cohort of European Prospective Investagation of Cancer and Nutrition (EPIC-Norfolk)," British Medical Journal, vol. 322, no. 7277, pp. $15-18,2001$.

[19] G.-C. Zhong, M.-X. Ye, J.-H. Cheng, Y. Zhao, and J.-P. Gong, "HbAlc and risks of all-cause and cause-specific death in subjects without known diabetes: a dose-response meta-analysis of prospective cohort studies," Scientific Reports, vol. 6, Article ID 24071, 2016.

[20] D. Sluik, H. Boeing, J. Montonen et al., "HbA 1c measured in stored erythrocytes is positively linearly associated with mortality in individuals with diabetes mellitus," PLOS ONE, vol. 7, no. 6, Article ID e38877, 2012. 
[21] Y. K. Cheon, J. K. Koo, Y. S. Lee, T. Y. Lee, and C. S. Shim, "Elevated hemoglobin Alc levels are associated with worse survival in advanced pancreatic cancer patients with diabetes," Gut and Liver, vol. 8, no. 2, pp. 205-214, 2014.

[22] K. Erickson, R. E. Patterson, S. W. Flatt et al., "Clinically defined type 2 diabetes mellitus and prognosis in early-stage breast cancer," Journal of Clinical Oncology, vol. 29, no. 1, pp. 54-60, 2011.

[23] W.-K. Xiao, D. Chen, S.-Q. Li, S.-J. Fu, B.-G. Peng, and L.-J. Liang, "Prognostic significance of neutrophil-lymphocyte ratio in hepatocellular carcinoma: a meta-analysis," BMC Cancer, vol. 14, no. 1, article 117, 2014.

[24] F. Ji, Y. Liang, S.-J. Fu et al., "A novel and accurate predictor of survival for patients with hepatocellular carcinoma after surgical resection: The neutrophil to lymphocyte ratio (NLR) combined with the aspartate aminotransferase/platelet count ratio index (APRI)," BMC Cancer, vol. 16, article 137, 2016.

[25] W. Liao, J. Zhang, Q. Zhu et al., "Preoperative neutrophil-tolymphocyte ratio as a new prognostic marker in hepatocellular carcinoma after curative resection," Translational Oncology, vol. 7, no. 2, pp. 248-255, 2014.

[26] G.-Q. Huang, G.-Q. Zhu, Y.-L. Liu et al., "Stratified neutrophilto-lymphocyte ratio accurately predict mortality risk in hepatocellular carcinoma patients following curative liver resection," Oncotarget, vol. 7, no. 5, pp. 5429-5439, 2016.

[27] H.-J. Yang, Z. Guo, Y.-T. Yang et al., "Blood neutrophil-lymphocyte ratio predicts survival after hepatectomy for hepatocellular carcinoma: a propensity score-based analysis," World Journal of Gastroenterology, vol. 22, no. 21, pp. 5088-5095, 2016.

[28] J. Zhang, L. Ye, J. Zhang et al., "MELD scores and childpugh classifications predict the outcomes of ERCP in cirrhotic patients with choledocholithiasis: a retrospective cohort study," Medicine (United States), vol. 94, no. 3, p. e433, 2015.

[29] K. A. Attia, K. C. Ackoundou-N'guessan, A. T. N'dri-yoman et al., "Child-Pugh-Turcott versus Meld score for predicting survival in a retrospective cohort of black African cirrhotic patients," World Journal of Gastroenterology, vol. 14, no. 2, pp. 286-291, 2008.

[30] B. Angermayr, M. Cejna, F. Karnel et al., "Child-Pugh versus MELD score in predicting survival in patients undergoing transjugular intrahepatic portosystemic shunt," Gut, vol. 52, no. 6, pp. 879-885, 2003. 


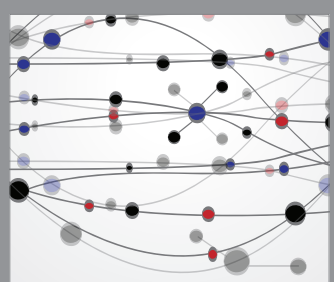

The Scientific World Journal
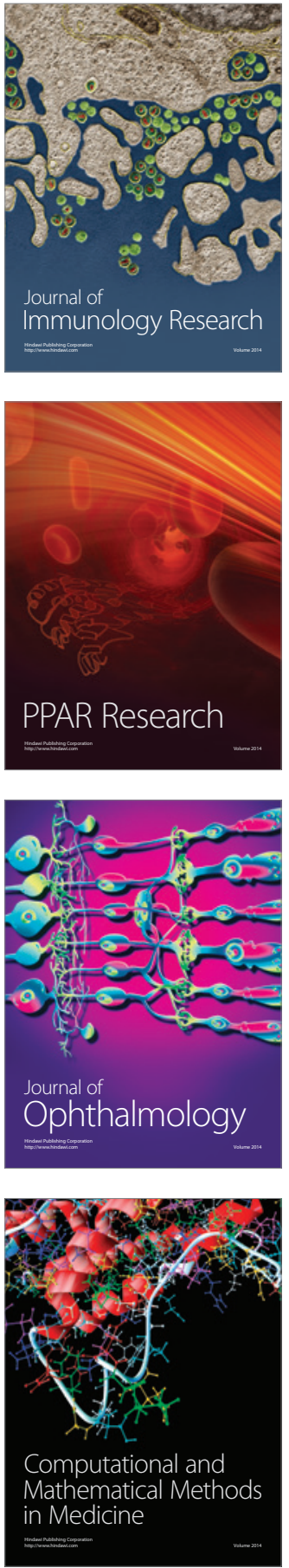

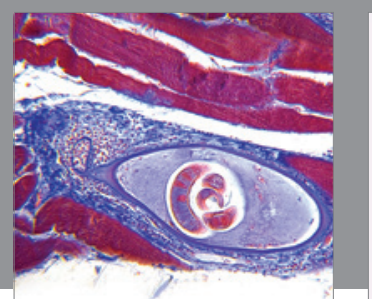

Gastroenterology Research and Practice
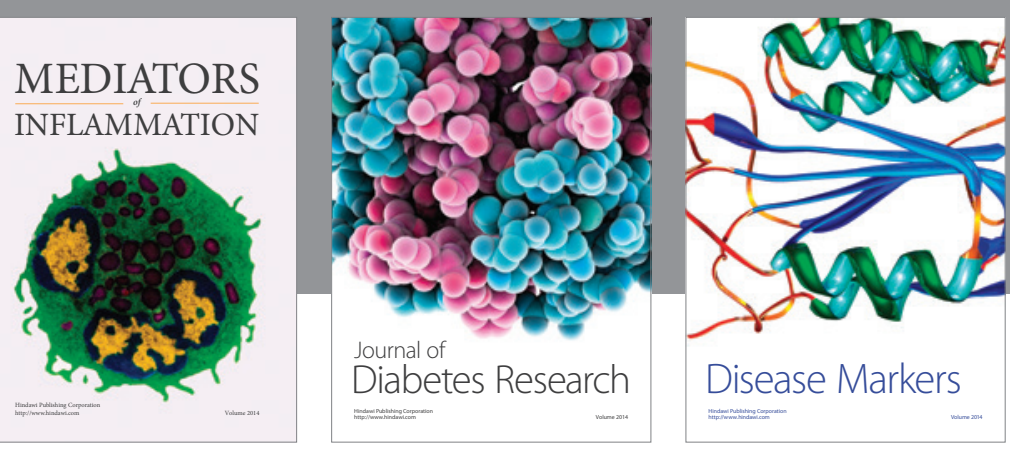

Disease Markers

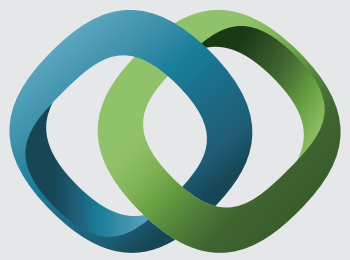

\section{Hindawi}

Submit your manuscripts at

https://www.hindawi.com
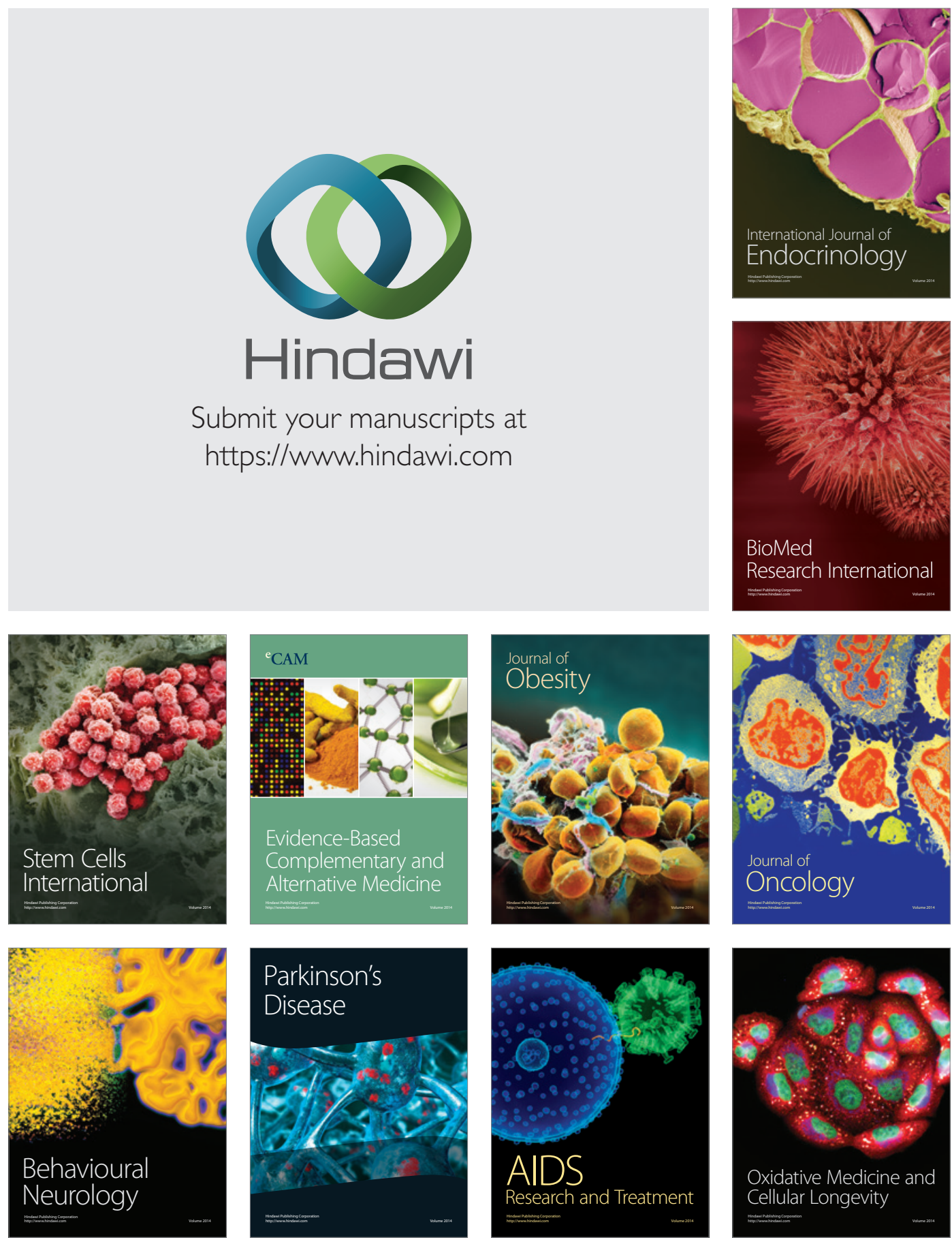